\title{
miR-20a attenuates acute lung injury in septic rats via targeting TLR4
}

\author{
Shuping $\mathrm{Li}^{1}$, Yang Sun ${ }^{1}$, Xingming Tang ${ }^{2}$, Li Wang ${ }^{2, *}$, Xiaoyuan Cheng ${ }^{1}$
}

${ }^{1}$ Department of Emergency, the First Affiliated Hospital of Chengdu Medical College, 610000 Chengdu, Sichuan Province, China

${ }^{2}$ Department of Anesthesiology, the First Affiliated Hospital of Chengdu Medical College, 610000 Chengdu, Sichuan Province, China

\section{*Correspondence}

wangli_88668@163.com

(Li Wang)

\begin{abstract}
Background: Sepsis is most likely to cause lung damage in patients, and the detection rate and mortality rate are high. Here, we investigated the expression of miR-20a in sepsis-induced acute lung injury (ALI) rats and its effect on inflammatory response, and reveal its possible molecular mechanism.

Method: The model of acute lung injury caused by sepsis in rats was established by cecal ligation and puncture. The expression of miR-20a in lung tissue was determined by RTqPCR. Acute lung injury rats were injected with $5 \mathrm{nmol}$ miR-20a agomir or agomir NC every day for 3 days. Rats were sacrificed by arterial bleeding and lung tissues were removed. Serum interleukin (IL) $-1 \beta$, IL-6, and tumor necrosis factor alpha (TNF- $\alpha$ ) were detected by ELISA. HE staining was used to observe the pathology of lung tissue and calculate the pathological score of lung injury. Western blot to determine the level of TLR4 and nuclear transcription factor $\kappa \mathrm{B}$ p65 (NF- $\kappa \mathrm{B}$ p65) protein in lung tissue. The luciferase reporter assay was used to verify the binding effect of miR-20a on the 3 non-coding TLR4.

Results: We found that compared with that in Normal group, the expression of miR-20a in lung tissues of rats with ALI was decreased $(p<0.05)$. In miR-20a agomir group, the plasma level of IL-1 $\beta$, IL-6, and TNF- $\alpha$ was significantly lower than that in agomir $\mathrm{NC}$ group and ALI group $(p<0.05)$, while higher than those in Normal group $(p<$ $0.05)$. The HE staining results showed that the pathological score of lung injury in rats in miR-20a agomir group was lower than that of agomir NC group and ALI group $(p<$ 0.05 ). Compared with agomir NC group and ALI group, the expression of TLR4 and NF- $\kappa \mathrm{B}$ p65 in miR-20a agomir group was decreased $(p<0.01)$. The luciferase reporting experiment confirmed that TLR4 was a target gene of miR-20a.

Conclusion: To sum up, miR-20a exerts a protective effect on sepsis-induced ALI rats through its anti-inflammatory effect. The targeting of TLR4 by miR-20a may be an effective method to reduce the inflammatory response in sepsis-induced ALI.
\end{abstract}

\section{Keywords}

Sepsis; Acute lung injury; miR-20a; TLR4

\section{Introduction}

Sepsis is a systemic inflammatory response caused by infectious factors, which often leads to multiple organ dysfunction when it develops to a serious stage. As a most vulnerable target organ in sepsis, lung injury is the earliest and the highest incidence in patients with sepsis, and it is also one of the main causes of death in patients with sepsis [1]. Current studies have shown that inflammatory response is the key molecular mechanism of sepsis-induced ALI, and inhibition of inflammatory response in patients with sepsis plays a protective effect on ALI [2-4]. As an inflammation related miRNA, miR-20a plays a negatively regulatory role in the immune process of the body [5]. However, little study regarding whether miR20a is involved in sepsis-induced ALI. This study aims to investigate the expression of miR-20a in sepsis-induced ALI and its effect on inflammatory response, and to clarify the underlying molecular mechanism.

\section{Materials and methods}

\subsection{Main reagents}

293T cells were from Yuchi Biotechnology Co., Ltd (Shanghai, China). Developer, protein double staining marker, Trizol reagent and one-step reverse transcription fluorescence quantitative kit were purchased from Shanghai Shenggong Biotechnology Co., Ltd (Shanghai, China). TLR4 (Abcam, Cambridge, MA, UK, ab13867). NF- $\kappa$ B p65 (Abcam, ab16502) and GAPDH (Abcam, ab9485) antibodies, HRP labeled second antibody (Abcam, ab6721), IL-1 $\beta$ (Abcam, ab216165) 
ELISA reagent, IL-6 (Abcam, ab100712) ELISA reagent and TNF- $\alpha$ (ab208348) ELISA reagent were all purchased from aibokang Trading Co., Ltd (Shanghai, China). The dual luciferase detection and reporting system kit, miR-20a mimics, miR-20a agomir and their respective negative controls were purchased from Promega Corporation, Madison, USA.

\subsection{ALI rats model}

SPF-grade Sprague-Dawley (SD) rats (male, 4-5 weeks old, 80-100 g) were provided by the Laboratory Animal Science and Technology Center of Jiangxi University of Traditional Chinese Medicine (animal certificate number SCXK. Rats were raised in the Laboratory Animal Science Center of Nanchang University. ALI rats model was constructed as described by Rittirsch et al.'s [6] method. Briefly, in order to trigger a systemic inflammatory response, the cecal ligation and puncture model was conducted, then, bacteria were translocated into the blood compartment. The lung tissue in rats have obvious pathological damages such as destruction of alveolar wall integrity, congestion, edema, thickening, and inflammatory cell infiltration, which is considered as successful models of ALI. All animal experiments were approved by the Ethics Committee of the First Affiliated Hospital of Chengdu Medical College (Approval No 2019-013) [7].

\subsection{Injection and grouping}

Thirty successful rats model were randomly divided into three groups (10 rats in each group): (1) ALI group: pleural injection of PBS buffer; (2) Agomir NC group: pleural injection of Agomir NC (meaningless sequence); (3) miR-20a agomir group: miR-20a agomir was injected into the chest cavity. The rats were injected for three consecutive days, and the injection dose was referred to the literature [8]. On the 4th day, the rats were sacrificed by arterial bloodletting and lung tissue was removed. In addition, 10 rats of the same age were selected as normal control group.

\subsection{RT-qPCR}

The expression of miR-20a in different groups was detected by RT-qPCR. RNA was extracted from the lung tissues of rats by Trizol, and the expression of miR-20a was detected according to the instructions of the one-step reverse transcription fluorescence quantitative kit. miR-20a, forward primer 5'-CGGCGCTAAAGTGCTTATAGTGC-3' and reverse primers 5'-ATCCAGTGCAGGGTCCGAGG-3'; U6, forward primer 5'-GCTTCGGCAGCACATATACTAAAAT-3, reverse primers 5'-CGCTTCACGAATTTGCGTGTCAT-3'.

\subsection{ELISA}

The expression levels of inflammatory cytokines (IL-1 $\beta$, IL6 and TNF- $\alpha$ ) were detected by ELISA. The blood was centrifuged (3000 r/min, $5 \mathrm{~min}$ ), and the plasma was collected and used for analysis. The procedure was carried out based on the instructions of ELISA detection kit, and the standard curve was drawn for determination.

\subsection{HE staining}

The lung tissues were collected $(1.5 \mathrm{~cm} \times 1.5 \mathrm{~cm} \times 1.4$ $\mathrm{cm}$ ), fixed by $10 \%$ formaldehyde and stained by $\mathrm{HE}$ after paraffin embedding. The pathological score of lung injury was obtained as described previously [9]: (1) edema, (2) inflammatory cell infiltration, and (3) tissue hemorrhage. Each item has a minimum score of 0 and a maximum score of 4 . Among them: no damage, count for 0; slight damage (about $25 \%$ bleeding range), count for 1 ; moderate damage (about $50 \%$ bleeding range), count for 2 ; severe damage (about $75 \%$ bleeding range), count for 3 ; the most serious damage (about the whole field of vision bleeding), count for 4 . The total score of the degree of lung injury was the total score of each pathological score of lung injury.

\subsection{Western blot}

The protein level was detected by Western blot. The lung tissues of each group were added with RIPA lysate, lysed on ice (20 minutes), centrifuged $\left(4^{\circ} \mathrm{C}, 13000 \mathrm{rpm}, 20 \mathrm{mins}\right)$, and the supernatant was finally taken and the protein content was determined using the BCA kit. The protein solution $(35 \mu \mathrm{g} / 30$ $\mu \mathrm{L}$ ) was used for SDS-PAGE, transferred to membrane and blocked with blocking solution (2\% BSA). Subsequently, the primary antibody (with GAPDH antibody as reference) was added ( $4{ }^{\circ} \mathrm{C}$, overnight). After that, the secondary antibody was added and incubated at room temperature for $1 \mathrm{~h}$. After ECL exposure, the Alpha Imager HP image documentation system (Alpha Innotech, San Leandro, CA) was used for analyzing the results.

\subsection{Luciferase reporter assay}

Luciferase reporter assay was performed to investigate the binding sites between miR-20a and TLR4. The wild-type (WT) and mutant (MUT) TLR4 3'-UTR dual fluorescent reporter plasmids were firstly constructed. The $293 \mathrm{~T}$ cells in logarithmic growth phase were seeded into 12-well cell plates ( $10^{5}$ cells/well), and the TLR4-WT, TLR4-MUT and miR-20a mimics or mimics NC negative controls were co-transfected into 293 T cells. Each experiment was replicated for 6 times. After $48 \mathrm{~h}$ of incubation, the luciferase activity was detected according to the dual luciferase reporter gene detection kit.

\subsection{Statistics}

All data were expressed as mean \pm standard deviation (SD) and analyzed by using SPSS 21.0 software. With normal distribution, the independent sample $t$ test was used for comparion between two groups, and the one-way ANOVA was used for comparison among multiple groups $(\alpha=0.05)$. $p$ value less than 0.05 was considered statistically significant.

\section{Results}

\subsection{Expression of miR-20a in the lung tissue from sepsis-induced ALI rats}

RT-qPCR results (Fig. 1) showed that the relative expression of miR-20a $(2.03 \pm 0.06)$ in the lung tissue from sepsisinduced ALI rats was significantly lower than that from normal 
rats $(10.05 \pm 0.49)(\mathrm{t}=16.29, p<0.001)$. To explore the functional role of miR-20a in the progression of ALI, miR-20a was overexpressed in ALI rats. The expression of miR-20a in the lung tissue from rats in the miR-20a agomir group (5.03 \pm $0.17)$ was significantly up-regulated as compared with that in the agomir NC group $(2.02 \pm 0.06)(\mathrm{t}=16.50, p<0.001)$.

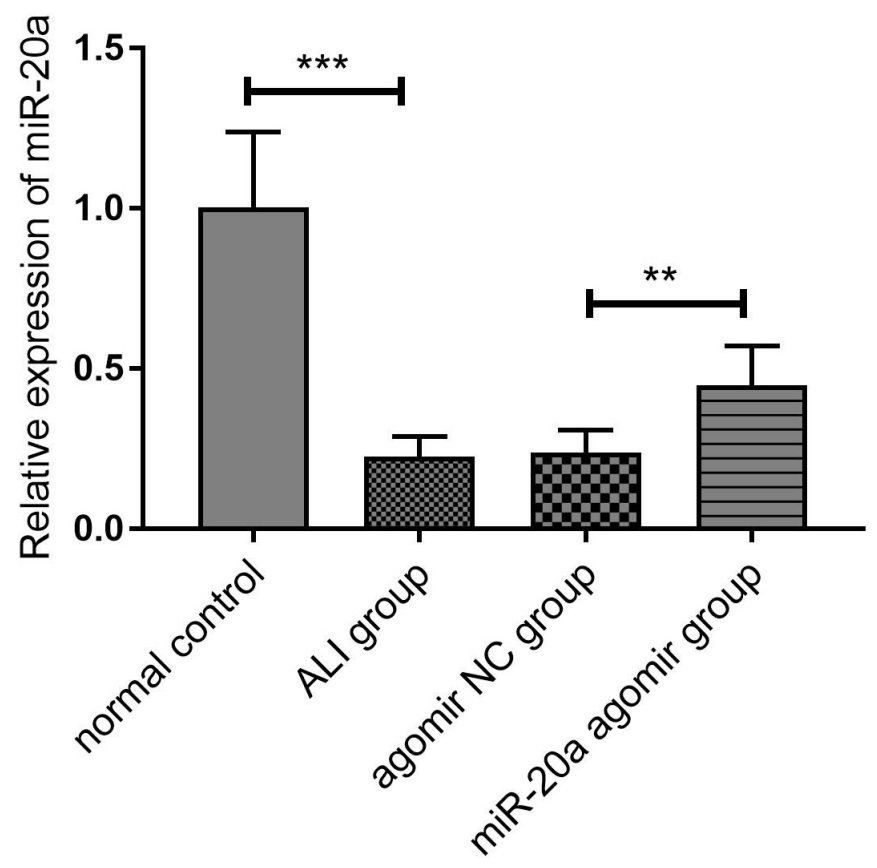

F I GURE 1. The expression of miR-20a in different groups. qRT-PCR was used to detect the expression of miR20a in ALI, miR-20a agomir group and their controls. $\mathrm{n}=6$ in each group. Data were expressed as mean \pm SD. $* * p<0.01$ and $* * * p<0.001$ represent statistically difference.

\subsection{Effects of miR-20a agomir on the expression of IL-1 $\beta$, IL- 6 and TNF- $\alpha$ in plasma from sepsis-induced ALI rats}

The expression levels of IL- $1 \beta$, IL- 6 and TNF- $\alpha$ in plasma were significantly elevated in sepsis-induced ALI rats as compared with that in normal control $(p<0.05)$, suggesting an inflammatory response. However, the level of these cytokines was significantly reduced in the plasma from ALI rats after injection of miR-20a agomir compared with agomir NC group $(p<0.05)$, indicating that the expression of these factors can be suppressed by miR-20a. Detailed information was shown in Table 1.

\subsection{Effect of miR-20a agomir on the histological changes from sepsis-induced ALI rats}

HE staining results showed that the structure of alveoli and bronchus in the normal control group was clear and complete, and there was no inflammatory cell infiltration and exudate. In the lung tissue of ALI group and agomir NC group, the alveolar septum was thickening, the obvious dilatation of blood vessels was found in the interstitium, and a large number of inflammatory cells in the lung interstitium around the bronchus were infiltration, and the alveolar cavity was filled with serous exudate. In the miR-20a agomir group, the pathological injury of lung tissue was mild, and a small amount of inflammatory cell infiltration and exudate were observed (Fig. 2). The lung injury score in miR-20a agomir group, agomir NC group, ALI group and normal control was $3.40 \pm 0.34,9.60 \pm 0.31,9.70$ \pm 0.26 and $0.50 \pm 0.22$, respectively. Taken together, the pathological score of lung injury in miR-20a agomir group was lower than that in agomir NC group and ALI group $(p<0.05)$, but higher than that in normal control $(p<0.05)$, indicating the protective effects of miR-20a agomir on lung tissue.

\subsection{Effect of miR-20a agomir on the expression of TLR4 and NF- $\kappa$ B p 65 in lung tissue from sepsis-induced ALI rats}

The protein expression levels of TLR4 and NF- $\kappa \mathrm{B}$ p65 in lung tissue were significantly up-regulated in miR-20a agomir group compared with that in control group (Fig. 3, $p<0.05$ ), which were significantly reduced as compared with that in the NC group and ALI group $(p<0.05)$. These results indicated that miR-20a agomir could induce the expression of TLR4 and $\mathrm{NF}-\kappa \mathrm{B}$ p65 in lung tissue from sepsis-induced ALI rats.

\subsection{The direct relationship between miR-20a and TLR4}

The binding site between miR-20a and TLR4 was predicted by Targetscan bioinformatics website. The sequence was shown in the left panel of Fig. 3, and the results of luciferase assay showed that in TLR4 wild type 3'-UTR, the relative activity of luciferase in miR-20a mimics group was significantly lower than that in mimics NC group ( $p<0.001)$. However, in TLR4 mutant 3'-UTR, the relative activity of luciferase in miR-20a mimics group did not show any change as compared with mimics NC group (Fig. 4, right panel). These results proved that miR-20a could directly target the 3'-UTR of TLR4.

\section{Discussion}

As one of small RNAs, miRNA has been reported related to the occurrence and development of tumor [10], cardiovascular disease [11], diabetes [12], human genetic disease [13] and nervous system disease [14]. Wu Songlin et al. [15] showed that miR-125b was significantly decreased in lipopolysaccharide (LPS)-induced ALI rats, and was negatively correlated with the expression of TNF- $\alpha$ and IL-6. Leng et al. [15] found that miR-483-5p was up-regulated in the lung tissue of sepsis-induced ALI mice. By inhibiting the expression of protein inhibitor activating STAT1 (PIAS1), miR-483-5p could promote the inflammatory response and induce the apoptosis of pulmonary microvascular endothelial cells and thus aggravated ALI. Therefore, miRNA may play a key role in the occurrence and development of ALI.

miR-20a, one of the members of miR-17/92 cluster, is located on chromosome $13 \mathrm{q} 31.1$ and mainly participates in inflammatory response [5]. Li et al. [16] pointed out that overexpression of miR-20a may target the regulation of thioredoxin interacting protein (TXNIP) and reduce the activity of NLRP3. Chen et al. [17] have shown that miR-20a 
TA B L E 1. Effect of miR-20a agamir on the levels of IL-1 $\beta$, IL-6 and TNF- $\alpha$ in plasma from sepsis-induced ALI rats.

$\begin{array}{lcccc}\text { Project } & \text { miR-20a agomir group } & \text { Agomir NC group } & \text { ALI group } & \text { Normal control } \\ \text { IL-1 } \beta(\mathrm{ng} / \mathrm{L}) & 85.65 \pm 0.63^{* \# \Delta} & 143.20 \pm 0.52 & 144.20 \pm 0.66 & 74.12 \pm 0.90 \\ \text { IL-6 }(\mu \mathrm{g} / \mathrm{L}) & 0.60 \pm 0.01^{* \# \Delta} & 0.87 \pm 0.01 & 0.88 \pm 0.01 & 0.50 \pm 0.01 \\ \text { TNF- } \alpha(\mu \mathrm{g} / \mathrm{L}) & 0.30 \pm 0.01^{\text {*\# }} & 0.73 \pm 0.01 & 0.72 \pm 0.01 & 0.20 \pm 0.01\end{array}$
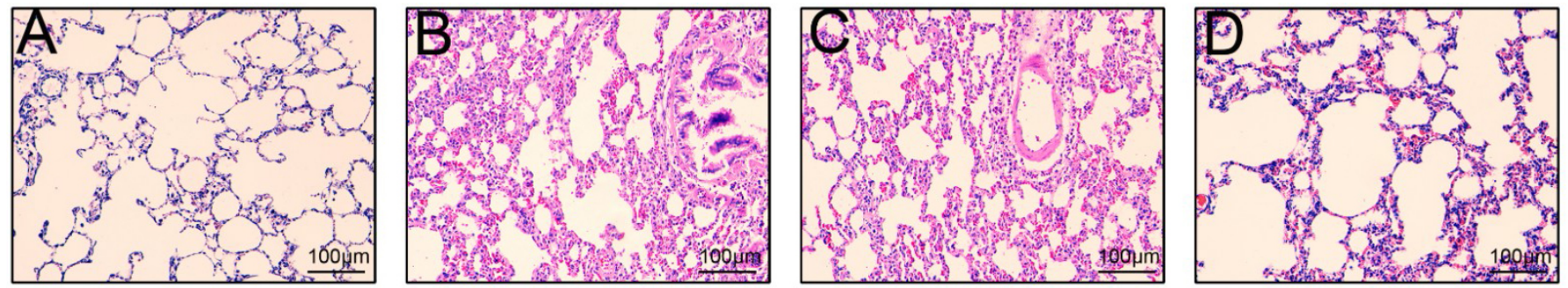

F I G U R E 2. Effect of miR-20a on pathological morphology of lung tissue $(\mathbf{H E} \times \mathbf{2 0 0})$. The lung tissue of rats with sepsis was fixed with $10 \%$ formaldehyde, and the pathological changes were observed by HE staining after paraffin embedding. The lung tissue appearance of rats in normal control (A), ALI group (B), agomir NC group (C) and miR-20a agomir group (D). $\mathrm{n}=6$ in each group. Data were expressed as mean $\pm \mathrm{SD}$.

A
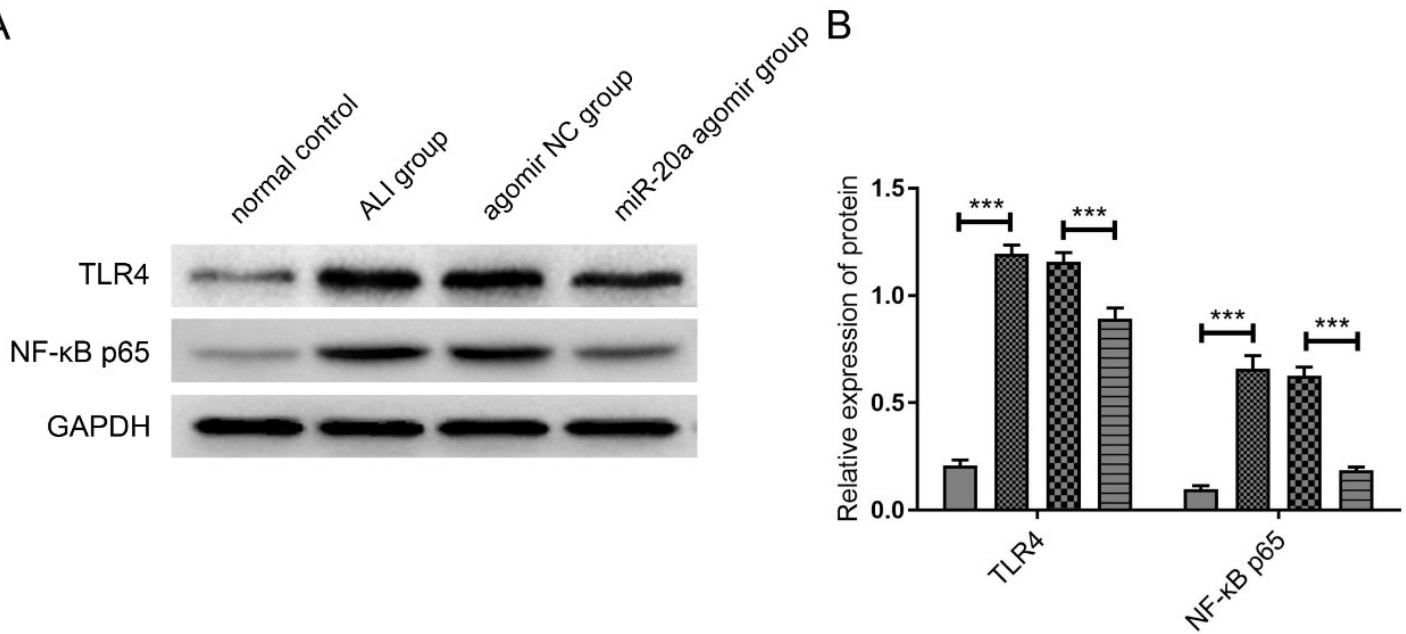

$\square$ normal control

ALI group

agomir NC group

$\square$ miR-20a agomir group

F I G U R E 3. miR-20a inhibits the expression of TLR4 and NF- $\kappa$ B p65 in rat lung tissue. (A) The expressions of TLR4, NF- $\kappa$ B p65 and GAPDH in normal control, ALI group, agomir NC group and miR-20a agomir group were detected by Western blot. (B) Alpha Imager HP gel imaging system was used to analyze the relative protein expression levels of TLR4 and NF- $\kappa$ B p65 in lung tissues in four groups. $\mathrm{n}=6$ in each group. Data were expressed as mean \pm SD. ${ }^{* *} p<0.01$ and ${ }^{* * *} p<0.001$ represent statistically difference.

could reduce inflammation by regulating TLR4 and TXNIP signaling pathways, thereby reducing the damage of human aortic endothelial cells (HAECs) induced by oxidized lowdensity lipoprotein (ox-LDL). Zhu et al. [18] pointed out that miR-20a could regulate macrophage infiltration, phagocytosis and pro-inflammatory cytokine secretion by targeting signal regulated protein $\alpha 1$ (SIRP $\alpha$ ). However, little study regarding whether miR-20a is involved in sepsis-induced ALI. This study found that miR-20a expression in the lung tissue of sepsisinduced ALI rats was decreased. Therefore, we speculated that miR-20a may play a role in the pathological process of ALI. To further explore the role of miR-20a in ALI, miR20a agomir was injected into sepsis-induced ALI rats, and the results showed that the levels of IL- $1 \beta$, IL-6, and TNF- $\alpha$ in serum were significantly reduced, and the pathological score of lung injury was significantly decreased. It is further confirmed that miR-20a plays a protective role in sepsis-induced ALI rats through its anti-inflammatory effect.

Toll like receptor 4 (TLR4) is an innate immune receptor, which is involved in immune regulation and inflammatory response. The inflammatory response caused by the activation of TLR-4 signaling pathway is the key molecular mechanism of sepsis-induced ALI. In particular, TLR4, MyD88, NF$\kappa \mathrm{B}, \mathrm{TNF}-\alpha$, IL- $1 \beta$, IL- 6 and other inflammatory mediators are the most studied molecules in TLR-4 signaling pathway, and are also considered as the targets of most drugs for the intervention of sepsis-induced ALI [19]. Liu Hui et al. [20] also showed that TLR4 inhibitor can suppress the inflammatory response mediated by $\mathrm{NF}-\kappa \mathrm{B}$ signaling pathway in septic rats and play a therapeutic role in the progression of 
A

TLR4 wild-type 3'-UTR:5' gaCUGAACUGGGUGUU CACUUU u 3'

miR-20a : 3' gaugGACGUGAUUC GUGAAA u 5'

TLR4 mutant 3'-UTR : 5' gaCUGAACUGGGUGUU GUGAAA u 3'

B

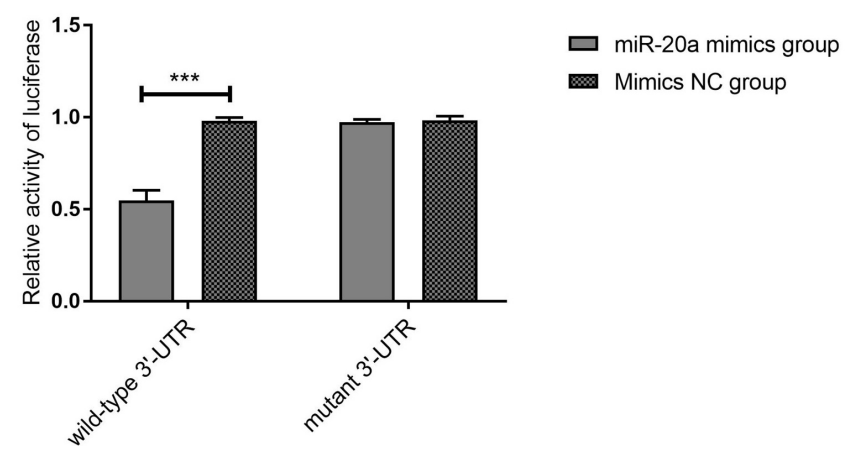

F I G U R E 4. Targeting relationship between miR-20a and TLR4. (A) The TargetScan bioinformatics site was used to predict the binding sites between miR-20a and TLR4. (B) The relative activity values of luciferase were detected using the dual luciferase reporter assay kit. $\mathrm{n}=6$ in each group. Data were expressed as mean $\pm \mathrm{SD} .{ }^{* * *} p<0.001$ represent statistically difference.

ALI. Through bioinformatics website, it was predicted that miR-20a could complement the 3'-UTR region of TLR4. In this study, the dual luciferase assay was used to verify the targeted regulatory relationship between miR-20a and TLR4. In addition, miR-20a agomir reduced the expression of TLR4 and NF- $\kappa \mathrm{B}$ p 65 , and reduced the inflammatory response in ALI rats. Taken together, it can be inferred that miR-20a can inhibit the inflammatory response in the progress of ALI by blocking the NF- $\kappa$ B pathway via targeting TLR4, which was similar to the results from $\mathrm{Li}$ [21]. Their results showed that miR-20a inhibited the production of inflammatory cytokines in LPS stimulated human gingival fibroblasts by activating the TLR4 signaling pathway.

This study demonstrated the effect of miR-20a on inflammatory response in sepsis-induced ALI was by inhibiting TLR4/NF- $\kappa \mathrm{B}$ signaling pathway. Based on the fact that one miRNA may regulate several target genes, the regulatory mechanisms of miRNA on target genes are complex. Therefore, further analysis on revealing the specific mechanism of miR-20a in sepsis ALI was needed.

\section{AUTHOR CONTRIBUTIONS}

SPL and YS designed the study, supervised the data collection. XMT analyzed the data, interpreted the data. LW and XYC prepare the manuscript for publication and reviewed the draft of the manuscript. All authors have read and approved the manuscript.

\section{ETHICS APPROVAL AND CONSENT TO PARTICIPATE}

Ethical approval was obtained from the Ethics Committee of the First Affiliated Hospital of Chengdu Medical College (Approval No 2019-013).

\section{ACKNOWLEDGMENT}

Thanks to all the peer reviewers for their opinions and suggestions.

\section{FUNDING}

This research received no external funding.

\section{CONFLICT OF INTEREST}

The authors declare no conflict of interest.

\section{REFERENCES}

[1] Xu Z, Huang Y, Mao P, Zhang J, Li Y. Sepsis and ARDS: the dark side of histones. Mediators of Inflammation. 2015; 2015: 205054.

[2] Zeng M, Sang W, Chen S, Chen R, Zhang H, Xue F, et al. 4-PBA inhibits LPS-induced inflammation through regulating ER stress and autophagy in acute lung injury models. Toxicology Letters. 2017; 271: 26-37.

[3] Li F, Chen Z, Tang H, Liang Y, Li Z, Wu J, et al. Protective effects of recombinant trichinella spiralis-53 000 protein combining with imipenem on polymicrobial septic mice. Zhonghua Wei Zhong Bing Ji Jiu Yi Xue. 2016; 28: 433-438. (In Chinese)

[4] Xu C, Chong L, Yu G, Zhang H. miR-574-5p alleviates sepsis-induced acute lung injury by regulating TRAF6/NF- $\kappa \mathrm{B}$ pathway. Tropical Journal of Pharmaceutical Research. 2020; 19: 676-682.

[5] Fu X, Qie J, Fu Q, Chen J, Jin Y, Ding Z. miR-20a-5p/TGFBR2 axis affects pro-inflammatory macrophages and aggravates liver fibrosis. Frontiers in Oncology. 2020; 10: 107.

[6] Rittirsch D, Huber-Lang MS, Flierl MA, Ward PA. Immunodesign of experimental sepsis by cecal ligation and puncture. Nature Protocols. 2009; 4: 31-36.

[7] Council NR. Guide for the care and use of laboratory animals (pp. 246). 8th edn. Washington, DC: The National Academies Press. 2011.

[8] Li X, Han J, Zhu H, Peng L, Chen Z. miR-181b-5p mediates TGF- $\beta 1$ induced epithelial-to-mesenchymal transition in non-small cell lung cancer stem-like cells derived from lung adenocarcinoma a549 cells. International Journal of Oncology. 2017; 51: 158-168.

[9] Xu T, Zhou L, Shi Y, Liu L, Zuo L, Jia Q, et al. Metabolomics approach in lung tissue of septic rats and the interventional effects of Xuebijing injection using UHPLC-Q-Orbitrap-HRMS. Journal of Biochemistry. 2018; 164: 427-435.

[10] Le Y, Chen T, Xun K, Ding T. Expression of the long intergenic noncoding RNA (lincRNA) of the NED25 gene modulates the microRNA$125 \mathrm{~b}$, STAT3, nitric oxide, and procalcitonin signaling pathways in patients with sepsis. Medical Science Monitor. 2018; 24: 4555-4566.

[11] Jokinen E. Obesity and cardiovascular disease. Minerva Pediatrica. 2015; 67: 25-32.

[12] Makaryus AN, McFarlane SI. Diabetes insipidus: diagnosis and treatment of a complex disease. Cleveland Clinic Journal of Medicine. 2006; 73: 65-71.

[13] Mikhail FM. Copy number variations and human genetic disease. Current Opinion in Pediatrics. 2014; 26: 646-652.

[14] McKinnon PJ. Genome integrity and disease prevention in the nervous system. Genes \& Development. 2017; 31: 1180-1194.

[15] Leng C, Sun J, Xin K, Ge J, Liu P, Feng X. High expression of miR-483-5p 
aggravates sepsis-induced acute lung injury. The Journal of Toxicological Sciences. 2020; 45: 77-86.

[16] Li X, Shen W, Sun Y, Li W, Sun Z, Liu Y, et al. MicroRNA-20a negatively regulates expression of NLRP3-inflammasome by targeting TXNIP in adjuvant-induced arthritis fibroblast-like synoviocytes. Joint Bone Spine. 2016; 83: 695-700.

[17] Chen M, Li W, Zhang Y, Yang J. MicroRNA-20a protects human aortic endothelial cells from Ox-LDL-induced inflammation through targeting TLR4 and TXNIP signaling. Biomedicine \& Pharmacotherapy. 2018; 103: 191-197.

[18] Zhu D, Pan C, Li L, Bian Z, Lv Z, Shi L, et al. MicroRNA-17/20a/106a modulate macrophage inflammatory responses through targeting signalregulatory protein $\alpha$. The Journal of Allergy and Clinical Immunology. 2013; 132: 426-436.e8.

[19] Qin L, Tan H, Wang Y, Xu C, Feng J, Li M, et al. Astragalus membranaceus and salvia miltiorrhiza ameliorate lipopolysaccharide-induced acute lung injury in rats by regulating the toll-like receptor 4/nuclear factor-kappa B signaling pathway. Evidence-Based Complementary and Alternative Medicine. 2018; 2018: 3017571.

[20] Liu J, Du J, Cheng X, Zhang X, Li Y, Fu X, et al. Effect of netrin-1 antiinflammatory factor on acute lung injury in sepsis rats. Medical Science Monitor. 2019; 25: 7928-7935.

[21] Li J, Wang M, Song L, Wang X, Lai W, Jiang S. LncRNA MALAT1 regulates inflammatory cytokine production in lipopolysaccharidestimulated human gingival fibroblasts through sponging miR-20a and activating TLR4 pathway. Journal of Periodontal Research. 2020; 55: $182-190$.

How to cite this article: Shuping Li, Yang Sun, Xingming Tang, Li Wang, Xiaoyuan Cheng. miR-20a attenuates acute lung injury in septic rats via targeting TLR4. Signa Vitae. 2021;17(4):157162. doi:10.22514/sv.2021.097. 